\title{
Parallel bimodal single-cell sequencing of transcriptome and chromatin accessibility
}

\author{
Qiao Rui Xing, ${ }^{1,2,11}$ Chadi A El Farran, ${ }^{1,3,11}$ Ying Ying Zeng, ${ }^{1,2}$ Yao Yi, ${ }^{1,3}$ \\ Tushar Warrier, ${ }^{1,3}$ Pradeep Gautam, ${ }^{1,3}$ James J. Collins, ${ }^{4,5,6}$ jian $\mathrm{Xu}_{1}{ }^{3,7}$ Peter Dröge, $^{2}$ \\ Cheng-Gee Koh, ${ }^{2} \mathrm{Hu} \mathrm{Li},{ }^{8}$ Li-Feng Zhang, ${ }^{2}$ and Yuin-Han Loh ${ }^{1,3,9,10}$
}

${ }^{1}$ Epigenetics and Cell Fates Laboratory, Institute of Molecular and Cell Biology, A*STAR, Singapore 138673, Singapore; ${ }^{2}$ School of Biological Sciences, Nanyang Technological University, Singapore 637551, Singapore; ${ }^{3}$ Department of Biological Sciences, National University of Singapore, Singapore 117558, Singapore; ${ }^{4}$ Institute for Medical Engineering and Science, Department of Biological Engineering, and Synthetic Biology Center, Massachusetts Institute of Technology, Cambridge, Massachusetts 02139, USA; ${ }^{5}$ Broad Institute of MIT and Harvard, Cambridge, Massachusetts 02142, USA; ${ }^{6}$ Wyss Institute for Biologically Inspired Engineering, Harvard University, Boston, Massachusetts 02115, USA; ${ }^{7}$ Department of Plant Systems Physiology, Institute for Water and Wetland Research, Radboud University, Heyendaalseweg 135, 6525 AJ, Nijmegen, The Netherlands; ${ }^{8}$ Center for Individualized Medicine, Department of Molecular Pharmacology \& Experimental Therapeutics, Mayo Clinic, Rochester, Minnesota 55905, USA; ${ }^{9}$ NUS Graduate School for Integrative Sciences and Engineering, National University of Singapore, Singapore 119077, Singapore; ${ }^{10}$ Department of Physiology, Yong Loo Lin School of Medicine, National University of Singapore, Singapore 119228, Singapore

\begin{abstract}
Joint profiling of transcriptome and chromatin accessibility within single cells allows for the deconstruction of the complex relationship between transcriptional states and upstream regulatory programs determining different cell fates. Here, we developed an automated method with high sensitivity, assay for single-cell transcriptome and accessibility regions (ASTARseq), for simultaneous measurement of whole-cell transcriptome and chromatin accessibility within the same single cell. To show the utility of ASTAR-seq, we profiled $384 \mathrm{mESCs}$ under naive and primed pluripotent states as well as a two-cell like state, 424 human cells of various lineage origins (B], K562, JK1, and Jurkat), and 480 primary cord blood cells undergoing erythroblast differentiation. With the joint profiles, we configured the transcriptional and chromatin accessibility landscapes of discrete cell states, uncovered linked sets of cis-regulatory elements and target genes unique to each state, and constructed interactome and transcription factor (TF)-centered upstream regulatory networks for various cell states.
\end{abstract}

[Supplemental material is available for this article.]

With the growing interest in understanding cellular heterogeneity, development of single-cell technologies has exploded in recent years. There are a multitude of single-cell techniques measuring genome (Navin et al. 2011; Wang et al. 2012; Gawad et al. 2014), transcriptome (Tang et al. 2009, 2010; Klein et al. 2015; Macosko et al. 2015), protein abundance (Huang et al. 2007; Hughes et al. 2014), cell surface protein (Stoeckius et al. 2017), copy number variation (Zong et al. 2012), DNA methylation (Guo et al. 2013; Lorthongpanich et al. 2013; Smallwood et al. 2014), chromatin accessibility (Buenrostro et al. 2015; Cusanovich et al. 2015; Jin et al. 2015; Pott 2017), immunoprecipitated chromatin (Rotem et al. 2015; Grosselin et al. 2019; Wang et al. 2019), chromatin architecture (Nagano et al. 2013; Flyamer et al. 2017; Ramani et al. 2017), and lineage tracing (Raj et al. 2018) at a single-cell resolution. These single-cell approaches allow for the deconstruction of cell types and cell states from mixed populations and the identification of cell state-specific genomic, transcriptomic, proteomic, and epigenomic programs.

However, unimodal single-cell techniques present limited values in uncovering intricate relationships across modalities,

\footnotetext{
${ }^{11}$ These authors contributed equally to this work.

Corresponding authors: yhloh@imcb.a-star.edu.sg, Li.Hu@mayo.edu Article published online before print. Article, supplemental material, and publication date are at http://www.genome.org/cgi/doi/10.1101/gr.257840.119. Freely available online through the Genome Research Open Access option.
}

which is of utmost importance to gain comprehensive understanding of cell states. To tackle this, multimodal single-cell methods have been developed for concurrent measurement of genome and transcriptome (Dey et al. 2015; Macaulay et al. 2015) and of transcriptome and DNA methylome within a single-cell (Angermueller et al. 2016; Cheow et al. 2016; Hu et al. 2016). With the technical advancement in the multi-omic fields, singlecell toolkits for joint profiling of transcriptome and chromatin accessibility are being developed to unravel the epigenetic mechanisms regulating gene transcription (Cao et al. 2018; Clark et al. 2018; Chen et al. 2019; Liu et al. 2019; Zhu et al. 2019; Xing et al. 2020). Among them, sci-CAR, SNARE-seq, and Paired-seq show high cell throughputs (tens of thousands), rendering them suitable for organism-scale measurements, such as cell atlas (Cao et al. 2018; Chen et al. 2019; Zhu et al. 2019). However, their high-throughput nature results in rare sequencing reads for each profiled cell and extensive loss of information (Xing et al. 2020). In comparison, scNMT-seq and scCAT-seq are applicable for studies with the intention of mining in-depth molecular distinctions between subpopulations (Clark et al. 2018; Liu et al. 2019). However, chromatin accessibility libraries prepared by scNMTseq suffer from extremely low mapping percentage and DNA

(C) 2020 Xing et al. This article, published in Genome Research, is available under a Creative Commons License (Attribution-NonCommercial 4.0 International), as described at http://creativecommons.org/licenses/by-nc/4.0/. 
mutations, which most likely resulted from the bisulfite treatment (Xing et al. 2020). Additionally, both methods involve separation of rare gDNA and RNA before any amplifications, which may result in significant loss of the lowly abundant genes and diploid genomic regions (Xing et al. 2020). Apart from this, this step also makes them less compatible with the automated platforms, thereby requiring remarkable manpower per experiment (Xing et al. 2020).

Here, we describe an automated technique with greater sensitivity, assay for single-cell transcriptome and accessibility regions (ASTAR-seq), which concurrently measures whole-cell transcriptome and epigenome accessibility within a single cell. To benchmark the technique, we applied it to deconstruct the heterogeneity of metastable mESCs under various cell states, human cell types of distinct lineage origins, and primary cells undergoing dynamic differentiation. We unraveled the cell state-specific transcriptomic and epigenetic programs, discovered unique pairs of cis-regulatory elements and putative target genes, and uncovered interactome pathways and upstream TFs associated with the heterogeneity observed.

\section{Results}

\section{Simultaneous measurement of transcriptome and chromatin accessibility within a single cell}

In the ASTAR-seq protocol, individual cells are first isolated at distinct cell capture sites linked with a series of reaction compartments on a Fluidigm C1 microfluidic chip (Fig. 1A). Open chromatin regions of each cell are then tagmented with Tn 5 transposase, during which accessible DNA regions (ATAC-DNA) are integrated with sequencing adaptors. Next, mRNA is reverse transcribed to double-stranded cDNA, which is then labeled with biotin during PCR amplification process. Biotinylation of cDNA enables its separation from ATAC-DNA using streptavidin beads. Lastly, the separated ATAC-DNA and cDNA fractions are further processed for library preparation and sequenced in parallel. The earlier prototype, in which reverse transcription was performed before transposition, was not successful (Supplemental Fig. S1A). This could be attributed to Tn5 transposase digesting singlestranded cDNA, which resulted in failure to separate cDNA from ATAC-DNA (Supplemental Fig. S1B-D). ASTAR-seq protocol was first optimized and tested on $1000 \mathrm{BJ}$ cells on benchtop. The optimal condition yielded abundant cDNA and achieved clear separation of ATAC-DNA and cDNA (Supplemental Fig. S1E-G).

As a proof of concept, we first applied ASTAR-seq to an ENCODE cell line, K562. Of 96 ASTAR ATAC-seq libraries sequenced, 92 libraries (95.8\%) passed the chromVAR QC thresholds (Fig. 1B; Supplemental Table 1). A median library size of 142,886 was detected, and $27.9 \%$ of fragments were in peaks, indicating high signal-to-noise of the ASTAR ATAC-seq libraries (Fig. 1B). Insert-size distribution of the ASTAR ATAC-seq libraries showed clear nucleosomal periodicity, a characteristic pattern of an ATAC-seq library (Fig. 1C; Buenrostro et al. 2015). In addition, ASTAR ATAC-seq libraries showed a Pearson's correlation of 0.946 with the published scATAC-seq libraries (Buenrostro et al. 2015), indicative of its high similarity to the unimodal libraries (Fig. 1D). On the other hand, 83 out of 96 ASTAR RNA-seq libraries $(86.5 \%)$ passed the QC thresholds set for gene detection rate $(\geq 15 \%)$ and exon mapping rate ( $\geq 75 \%)$ (Fig. 1E; Supplemental Table 1). A median of 4182 genes was detected (Supplemental Fig. S2A). In addition, scRNA-seq reads spread across the entire gene bodies, without biasing toward either ends of the mRNA
(Fig. 1F). Moreover, ASTAR RNA-seq showed a high Pearson's correlation $(R=0.87)$ with the published unimodal K562 scRNA-seq (Fig. 1G; Pollen et al. 2014).

To make a fair comparison with a similar bimodal technique scCAT-seq, we sequenced the K562 ASTAR-seq libraries at a comparable sequencing depth (40 single-cell libraries per lane of HiSeq 4000) (Supplemental Table 1). scCAT-seq libraries showed a significantly lower alignment rate to the human genome in comparison with ASTAR-seq (scATAC-seq: $67.6 \%$ vs. $85.8 \%$; scRNA-seq: $54.9 \%$ vs. $73.8 \%$ ) (Supplemental Fig. S2B). Additionally, a significantly higher percentage of ASTAR-seq libraries passed the QC thresholds for both the scATAC-seq and scRNA-seq than the scCAT-seq libraries (ASTAR-seq: 75.4\%; scCAT-seq: 43.2\%) (Supplemental Fig. S2C). For scATAC-seq libraries that passed QC, scCAT-seq and ASTAR-seq displayed comparable performance in terms of library complexity and signal-to-noise ratio (Fig. $1 \mathrm{H}$ ). On the other hand, comparable numbers of de-duplicated reads were detected for scRNA-seq libraries (scCAT-seq: 4,507,504; ASTAR-seq: $4,047,857)$, in line with their comparable sequencing depths (Fig. 1I), whereas ASTAR-seq detected 1014 more genes than scCAT-seq (ASTAR-seq: 9739; scCAT-seq: 8725) (Fig. 1I).

To comprehensively review other bimodal single-cell techniques, we systematically compared ASTAR-seq with scCAT-seq, sci-CAR, SNARE-seq, and Paired-seq in terms of profiled cells, QC rate, estimated cost per paired good-quality libraries, and QC matrices (Supplemental Fig. S2D-F). Among them, ASTAR-seq and scCAT-seq were of lower throughput, and ASTAR-seq showed the highest QC rate (Supplemental Fig. S2D). Correspondingly, owing to their high sequencing depth, ASTAR-seq and scCAT-seq displayed a higher cost per cell than the high-throughput methods (Supplemental Fig. S2E). Despite the comparable overall cost per experiment, the estimated cost per paired good-quality ASTAR-seq libraries is 2.1 times lower than that of scCAT-seq (Supplemental Fig. S2E). On the other hand, ASTAR-seq and scCAT-seq showed a significantly higher number of detected genes (approximately 10fold) and accessible sites (approximately 100-fold) than the highthroughput bimodal techniques (Supplemental Fig. S2F), whereas the compared techniques did not show specific trends in terms of signal-to-noise ratio (Supplemental Fig. S2F). Taken together, these data indicate the reliability of ASTAR-seq technique and show its superior performance in various aspects.

\section{Deconstruction of heterogeneity in mESCs under distinct cellular states}

We next applied ASTAR-seq to 192 E14 mESCs cultured in serum + LIF and $2 \mathrm{i}+$ LIF medium, which were named as mESCs and $2 \mathrm{i}$ cells throughout the study. All the sequenced scATAC-seq libraries passed the QC thresholds (Fig. 2A; Supplemental Table 2). scATAC-seq reads displayed an insert-size distribution with nucleosomal pattern and high enrichment at transcription start sites (TSSs) (Supplemental Fig. S3A,B). Moreover, these libraries showed a significantly higher signal-to-noise ratio than the unimodal mESC scATAC-seq libraries (Supplemental Fig. S3C; Buenrostro et al. 2015). Additionally, mESCs and $2 \mathrm{i}$ cells can be accurately distinguished by confusion matrix analysis based on their highly accessible regions (HARs) (Supplemental Fig. S3D). We then clustered mESCs and 2i ASTAR ATAC-seq libraries based on the overall accessibility profiles and accessibility of mouse JASPAR motifs (Fig. 2B; Supplemental Fig. S3E,F). mESCs and 2i cells were mostly clustered separately, but a certain degree of overlapping was observed (Fig. 2B; Supplemental Fig. S3F). Of note, chromatins

\section{Genome Research}

www.genome.org 

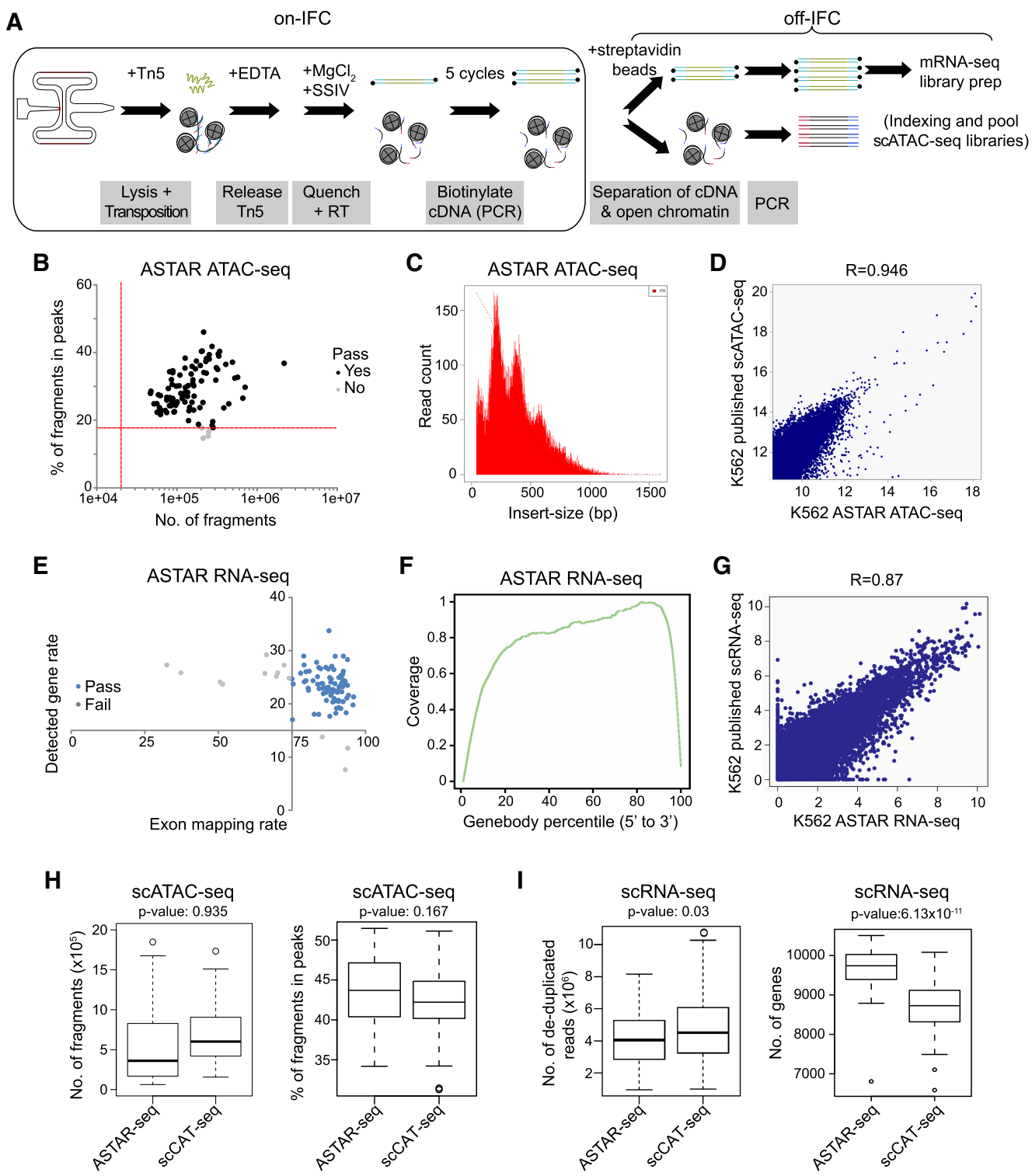

Figure 1. Assay for single-cell transcriptome and accessibility regions (ASTAR-seq). (A) Overview of ASTAR-seq protocol. (B) Dotplot revealing the percentage of fragments in peaks ( $y$-axis) against the number of fragments ( $x$-axis) of each K562 ASTAR ATAC-seq library. Red dotted lines represent the threshold values set for each criterion. (C) Histogram showing the frequency ( $y$-axis) of fragments with the indicated insert size ( $x$-axis). (D) Dotplot showing Pearson's correlation between K562 ASTAR ATAC-seq and the published K562 scATAC-seq libraries. ( $E$ ) Dotplot revealing detected gene rate $(y$-axis) of each K562 ASTAR RNA-seq library plotted against its exon mapping rate ( $x$-axis). Blue dots represent the libraries that pass QC, whereas gray dots represent the libraries of low quality. $(F)$ Line plot representing the coverage ratio ( $y$-axis) of K562 ASTAR RNA-seq reads over the gene bodies of housekeeping genes ( $x$-axis). (G) Dotplot showing Pearson's correlation between K562 ASTAR RNA-seq and the published K562 scRNA-seq libraries. $(H)$ Boxplots showing the number of fragments (left) and percentage of fragments in peaks (right) of sCATAC-seq libraries prepared by the scCAT-seq and ASTAR-seq protocol. Twotailed Student's $t$-test is used to calculate $P$-values. (I) Boxplots showing the number of de-duplicated reads (left) and genes (right) detected in scRNA-seq libraries prepared by the scCAT-seq and ASTAR-seq protocol. Two-tailed Student's $t$-test is used to calculate $P$-values.

containing motif sequences of KLF4, RARG, ZFX, KLF12, and MLXIP showed significant variability in terms of accessibility $(P$ value <0.05) (Supplemental Fig. S3E; Supplemental Table 2). For instance, KLF4 motif was highly accessible in $2 \mathrm{i}$ cells, whereas ZFX showed the opposite trend (Supplemental Fig. S3G). In the previous studies, not only was Klf4 reported to be up-regulated in mESCs under naive state compared with the primed state but also its overexpression facilitated cellular reprogramming of primed EpiSCs to naive ESCs (Guo et al. 2009; Jeon et al. 2016). Conversely, despite favoring self-renewal, mESCs with ZFX overex- pression failed to efficiently generate teratoma and chimera, which is in line with naive ESCs with low ZFX activity presenting high chimera formation rate (Galan-Caridad et al. 2007).

Likewise, the majority of mouse ASTAR RNA-seq libraries $(80.7 \%)$ passed the QC thresholds, in which a median of 2645 and 2429 genes were detected for mESCs and $2 \mathrm{i}$ libraries, respectively (Fig. 2C; Supplemental Fig. S3H; Supplemental Table 2). In addition, ASTAR RNA-seq libraries also showed full gene body coverage for the detected transcripts (Supplemental Fig. S3I). Moreover, mESC ASTAR-seq libraries highly correlated $(R=0.86)$ 
A

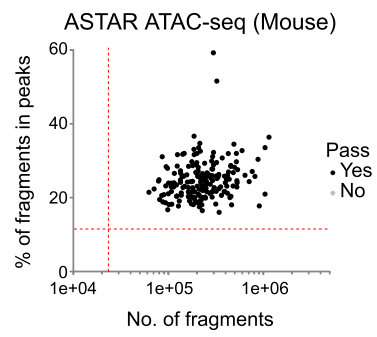

D

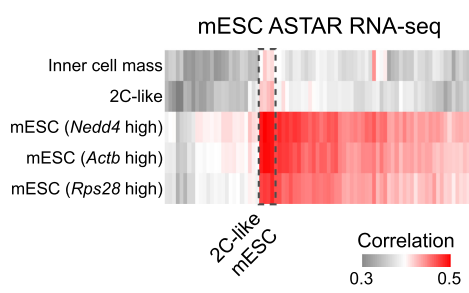

G

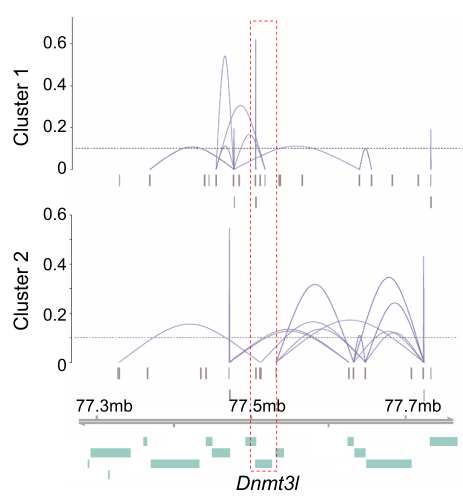

H

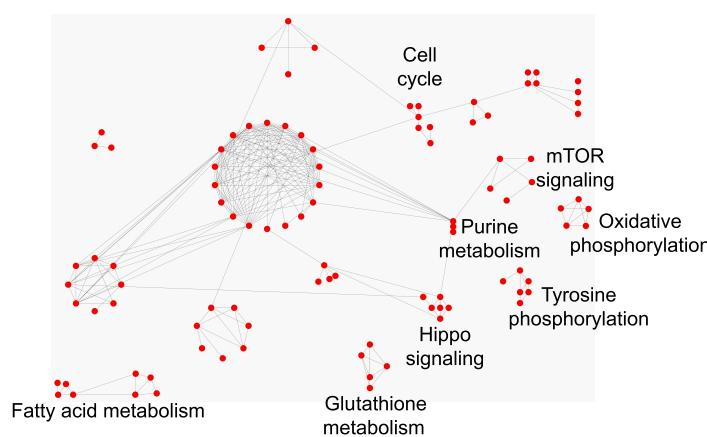

B

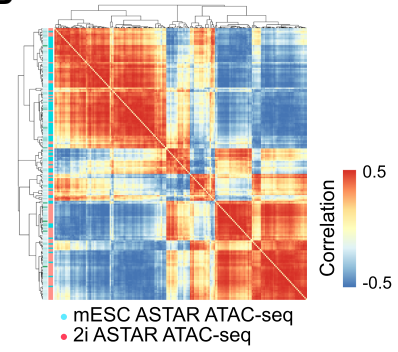

C

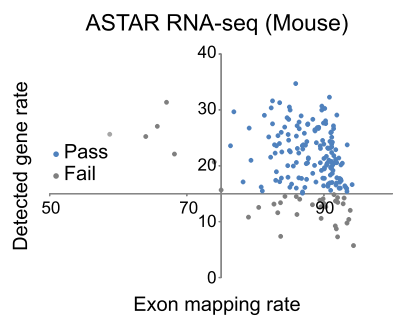

F ATAC-seq

E

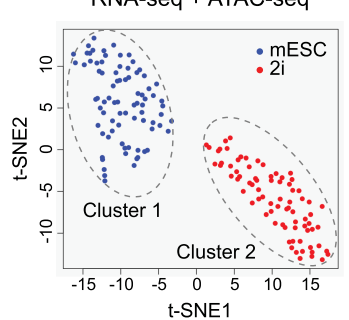

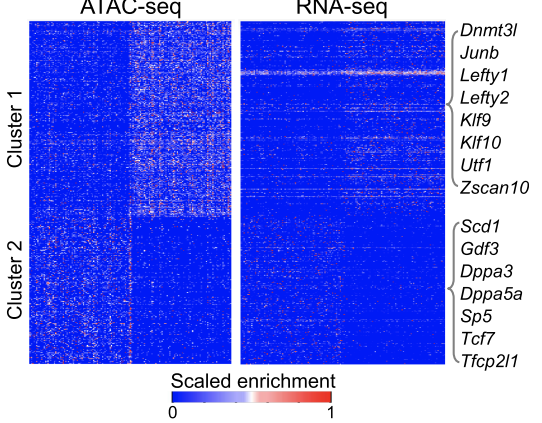
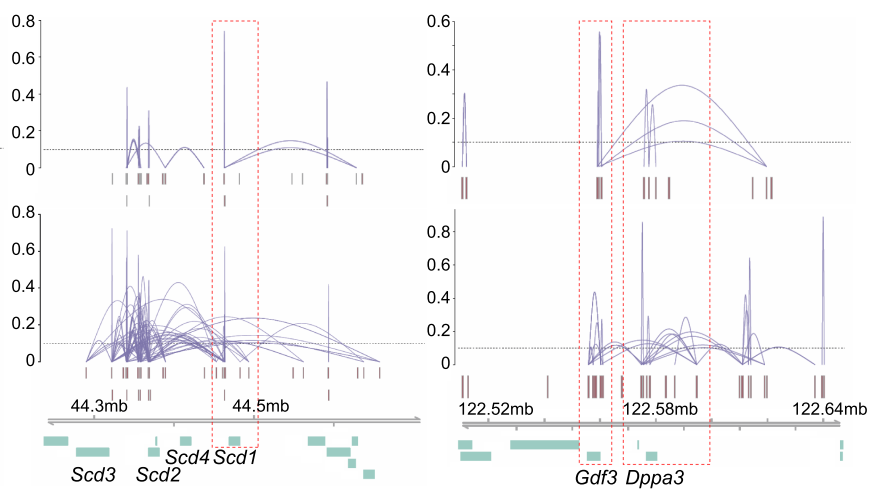

I
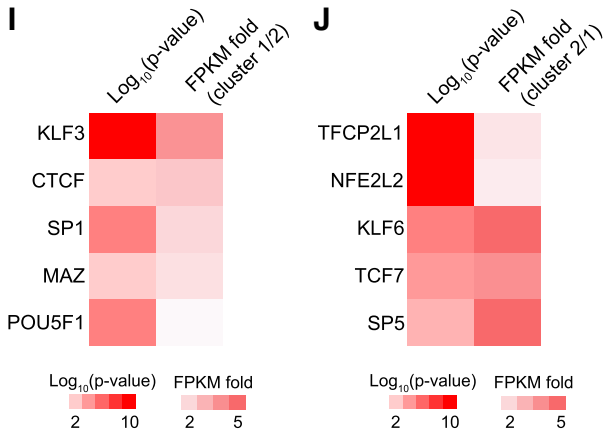

Figure 2. Transcriptomic and epigenetic heterogeneity within primed and naive mESCs. $(A)$ Dotplot revealing the percentage of fragments in peaks $(y$-axis) and number of fragments ( $x$-axis) of each mouse ASTAR ATAC-seq library. Red dotted lines represent the thresholds set for each criterion. (B) Heatmap showing the correlation among mESCs and 2i cells based on their ASTAR ATAC-seq libraries. (C) Dotplot revealing the detected gene rate ( $y$-axis) of each mouse ASTAR RNA-seq library plotted against its exon mapping rate ( $x$-axis). Blue dots represent the libraries that pass QC, whereas gray dots indicate the low-quality libraries. $(D)$ Heatmap revealing the correlation of each mESC ASTAR RNA-seq library ( $x$-axis) to various lineages of MCA panel ( $y$-axis). Color indicates the correlation level, ranging from gray (low) to red (high). Two-cell-like (2C-like) mESCs are boxed with a dotted line. ( $E$ ) NMF clustering of $\mathrm{mESC}$ s and $2 \mathrm{i}$ cells based on the correlative signals of their ASTAR ATAC-seq and ASTAR RNA-seq libraries. $(F)$ Heatmaps revealing pairs of accessible regulatory regions (left) and the corresponding target genes (right) that are differentially enriched between the NMF clusters. Each column represents a library, whereas each row represents a chromatin region (left) or a gene (right). Color indicates the accessibility (left) and expression (right) levels, ranging from blue (low) to red (high). Representative genes are indicated on the right. (G) Line plots showing the differential coaccessibility links between the highlighted regions and its surrounding regions, identified using Cicero. Top plots are constructed from ASTAR ATAC-seq libraries of cluster 1 cells, whereas bottom plots are constructed from that of cluster 2 cells. Peak heights ( $y$-axis) denote the coaccessibility scores. $(H)$ Interactome analysis revealing the top pathways enriched by cluster 2 -specific genes. $(I, J)$ Heatmaps showing the enrichment (left columns) of TF motifs on cluster 1-specific $(I)$ and cluster 2 -specific $(J)$ accessible regions and their relative expressions (right columns). 
with the published unimodal scRNA-seq libraries (Supplemental Fig. S3J; Svensson et al. 2017). Similarly, the confusion matrix illustrated that transcriptomic profiles detected by ASTAR-seq can accurately distinguish mESC and $2 \mathrm{i}$ cells (Supplemental Fig. S3K). To study the heterogeneity within mESCs, we correlated mESC ASTAR RNA-seq libraries to a mouse cell atlas (MCA) panel (Han et al. 2018). Analysis revealed three types of cells, including mESCs, ICM-like mESCs, and two-cell like (2C-like) mESCs, in agreement with a previous report (Fig. 2D; Macfarlan et al. 2012), whereas MCA analysis of 2i ASTAR RNA-seq libraries showed absence of a 2C-like population (Supplemental Fig. S3L). PCA also revealed a separate cluster of the minority 2C-like cells (Supplemental Fig. S3M). To confirm the presence of 2C-like cells in our mESCs culture, we used the 2C::tdTomato reporter constructed in the previous study (Macfarlan et al. 2012). Indeed, 1\%-2\% of mESCs consistently showed activation of the $2 \mathrm{C}$ reporter, which was significantly increased upon depletion of Ehmt2, a H3K9me3 methyltransferase, as previously described (Supplemental Fig. S3N; Macfarlan et al. 2012).

\section{Integrative analysis of ASTAR-seq uncovered cluster-specific cis-regulatory elements and their target genes}

To show the advantage of joint profiling, we then clustered mESCs and 2i ASTAR-seq libraries on the basis of bimodal data sets using coupled nonnegative matrix factorization (NMF) (Duren et al. 2018). Of note, two distinct clusters were always observed when clustering was based on either the differentially expressed genes or the differentially accessible regions that were identified by coupled NMF analysis (Supplemental Fig. S4A). Cluster 1 was mostly composed of mESCs, whereas cluster 2 was majorly composed of $2 \mathrm{i}$ cells. In comparison, joint clustering based on the NMF cluster-specific genes and accessible regions together showed superior performance in distinguishing the subpopulations, as seen from the clear and accurate separation of mESCs and $2 \mathrm{i}$ cells (Fig. 2E). In addition, correlation between accessibility and gene expression enabled us to identify regulatory networks specific to each NMF cluster (Fig. 2F; Supplemental Table 2). For instance, Dnmt3l, lefty1, $U t f 1$, and Zscan10 were highly accessible and expressed in cells of cluster 1, whereas $S c d 1$, Gdf3, Dppa3, Dppa5a, Sp5, and Tfcp2l1 were highly expressed in cluster 2 cells with greater accessibility (Fig. 2F; Supplemental Fig. S4B). These genes were previously reported to be important for naive and primed pluripotency, respectively. For example, higher expressions of Dnmt3l, lefty1, and Utf1 were observed for mESCs under naive state than primed state, whereas the opposite was observed for Dppa5a and Tfcp2l1 (Chen and Lai 2015; Choi et al. 2017; Ghimire et al. 2018). ZSCAN10 regulates epiblast-like cells through interaction with POU5F1, whereas SP5 facilitates the conversion of epiblast to naive ESCs (Buecker et al. 2014; Ye et al. 2016).

In addition to this, Cicero coaccessibility analysis (Pliner et al. 2018) showed that the Dnmt3l gene and its surrounding loci displayed open chromatin architecture with high interaction frequency in cluster 1 ; on the contrary, more $3 \mathrm{D}$ genomic interactions were observed for $S c d 1$, Gdf3, and Dppa3 in cluster 2 (Fig. 2G). The expression of Dnmt3l was reported to be reduced in naive ESCs, resulting in a global DNA demethylation (Leitch et al. 2013). On the contrary, SCD1 is a lipid metabolic enzyme involved in lipogenesis, and proteins associated with fatty acid metabolism were reported to be highly expressed in naive mESCs (Taleahmad et al. 2015, 2018). Similarly, Dppa3 and Gdf3 were known to be important for naive pluripotency (Chen et al. 2015; Sang et al. 2019). Altogether, this highlights the differential interactive networks in which the cluster-specific putative regulatory elements result in differential expression of the respective target genes.

\section{Identification of cluster-specific interactome pathways and TF-centered regulatory networks associated with naive and primed pluripotency}

NMF cluster-specific genes showed extensive interaction among themselves by involving in similar biological processes (Fig. $2 \mathrm{H}$; Supplemental Fig. S4C; Supplemental Table 2). Specifically, cluster 2 genes were majorly associated with metabolic pathways, including oxidative phosphorylation, glutathione metabolism, and fatty acids metabolism, which were reported to be key characteristics of naive pluripotency (Fig. 2H). For example, in terms of catabolism, naive ESCs show wide energy substrate usage through glycolysis, oxidative phosphorylation, and fatty acid oxidation, whereas primed ESCs mainly use glucose as energy source (Mathieu and Ruohola-Baker 2017). Glutathione is an antioxidant preventing cellular damage from the toxic molecules produced under oxidative stress during oxidative phosphorylation, which were found to be highly active in $2 \mathrm{i}$ cells compared with mESCs (Taleahmad et al. 2015, 2018). Similarly, anabolism processes, including amino acids and nucleotides synthesis, were reported to be highly active in the naive ESCs, which were also enriched in cluster 2-specific genes, such as tyrosine phosphorylation pathway and purine metabolism (Taleahmad et al. 2015, 2018).

Apart from the metabolic process, mTOR and Hippo signaling pathways were also enriched by the $2 \mathrm{i}$ cluster-specific genes (Fig. $2 \mathrm{H})$. Attenuation of Hippo signaling was observed along the developmental axis from early blastocyst to epiblast, whereas depletion of $Y A P / T A Z$, whose nuclear localization and regulatory function are repressed by Hippo signaling, caused differentiation specifically in mESCs but not in 2i cells (Lian et al. 2010; Azzolin et al. 2014; Weinberger et al. 2016; Hashimoto and Sasaki 2019). Likewise, proteins involved in the mTOR signaling pathway were highly expressed in naive ESCs, whereas disruption of Mtor led to early postimplantation lethality and prohibited ESCs development (Gangloff et al. 2004; Taleahmad et al. 2015). Mechanistically, mTOR positively regulated mitochondrial function and oxidative metabolism, which were highly active in naive ESCs ( $\mathrm{Yu}$ and Cui 2016). On the other hand, cluster 1 genes showed specific association with glucose metabolism and TGFB signaling (Supplemental Fig. S4C). TGFB/activin signaling was shown to be required for epiblast induction, whereas its inhibition resulted in conversion of epiblast to naive state (Han et al. 2011; Taleahmad et al. 2018).

There are also pathways commonly enriched for $2 \mathrm{i}$ and $\mathrm{mESC}$ clusters, such as ribosome complex, RNA splicing, and cell cycle (Fig. 2H; Supplemental Fig. S4C; Supplemental Table 2). However, the component genes of those complexes are distinct. For instance, genes related to the G1/S phase, such as Cdca3 and Mad2l1, were enriched in the 2i cluster (Supplemental Table 2), whereas genes associated with the G2M phase, including Cep76, Csnk1e, and Tubb3, were enriched in the cluster-specific genes of mESCs (Supplemental Table 2). They are in line with the prolonged G1/S phase and abbreviated G2M phases of naive mESCs compared with primed mESCs (Ter Huurne et al. 2017; Taleahmad et al. 2018).

To further examine the TFs responsible for the differential regulatory networks, we performed motif enrichment analysis for the cluster-specific accessible sites. KLF3, CTCF, SP1 and MAZ 
motifs were enriched on cluster 1-specific accessible regions, which were also highly expressed in cluster 1 cells (Fig. 2I; Supplemental Table 2). An earlier study reported lower genomic looping frequencies in $2 \mathrm{i}$ cells compared with mESCs (Krijger et al. 2016), supporting the higher CTCF regulon activity detected in cluster 1. On the contrary, motifs of TFCP2L1, NFE2L2, KLF6, TCF7, and SP5 were enriched on cluster 2-specific accessible regions, majority of which displayed higher expression in the cells of cluster 2 (Fig. 2J; Supplemental Table 2).

\section{ASTAR-seq recapitulates $2 \mathrm{C}$-like population in mESCs}

To further examine the capability of ASTAR-seq recapitulating known biology, we prepared ASTAR-seq libraries for mESCs in which DUX was overexpressed for $24 \mathrm{~h}$ in two batches (Supplemental Fig. S5). DUX overexpression was known to induce 2C-like fate in mESCs (Hendrickson et al. 2017). One hundred eighty-six out of 192 ASTAR ATAC-seq libraries passed QC, and a median of 2353 genes were detected in ASTAR RNA-seq libraries (Supplemental Fig. S5A,B). Of note, replicates of ASTAR-seq libraries showed distinctively high correlation between themselves in terms of both transcriptomic profile and chromatin accessibility $(R=0.987 ; 0.99)$ (Supplemental Fig. S5C). ASTAR RNA-seq libraries showed two clusters that were free of technical bias (Supplemental Fig. S5D). Cluster 2 showed a higher expression of genes associated with 2C-like fate, including Zscan4a, Zscan4c, Zscan4d, Zscan4f, Usp17la, Usp17lb, Usp17lc, and Eif4a3l1 (Supplemental Fig. S5E; Macfarlan et al. 2012; Hendrickson et al. 2017). Likewise, ASTAR ATAC-seq libraries distinguished different populations with no technical bias (Supplemental Fig. S5F). One population showed higher accessibility for DUX binding sites and MERVL loci (Supplemental Fig. S5G). This goes in agreement with the earlier studies reporting the reactivation of MERVL in 2C-like mESCs and the crucial role of DUX in maintaining 2C-like fate (Macfarlan et al. 2012; Hendrickson et al. 2017). Indeed, mESCs with a 2C-like transcriptomic signature showed a higher accessibility for MERVL loci and DUX binding sites (Supplemental Fig. S5H). Taken together, these data indicate the reproducibility and reliability of ASTAR-seq in recapitulating known biological phenomenon.

\section{Cell type-specific programs revealed by ASTAR-seq}

To expand the applicability, we also prepared ASTAR-seq libraries for various human cell lines, including BJ cells in adherent culture, and JK1 and Jurkat cells in suspension culture (Fig. 3). To characterize the molecular distinction among the hematopoietic cells, 96 K562 ASTAR-seq libraries sequenced at a similar depth were also included for the following analysis. Out of 384 libraries profiled, 375 ASTAR ATAC-seq libraries passed the QC thresholds for chromVAR (Supplemental Fig. S6A; Supplemental Table 3). In median, a library size of 55,193 was captured, and 35\% of fragments was in peaks (Supplemental Fig. S6A,B). Insert-size distribution of ASTAR ATAC-seq libraries also showed characteristic nucleosomal pattern of ATAC-seq libraries (Supplemental Fig. S6C; Buenrostro et al. 2015). In addition, ASTAR ATAC-seq libraries also showed a high similarity to the published scATAC-seq libraries $(R=0.897)$ (Supplemental Fig. S6D; Buenrostro et al. 2015). Confusion matrix analysis showed that the HARs detected by ASTAR-seq libraries can distinguish these cell lines with high accuracy (96.5\%) (Supplemental Fig. S6E). These indicate that the prepared ASTAR ATAC-seq libraries are of good quality.

Next, we clustered ASTAR ATAC-seq libraries based on enrichment of human JASPAR motifs and observed four clusters, among which BJ cells displayed distinct accessibility profiles and clustered away from the cells of blood lineage (Fig. 3A). Variability analysis identified TF motifs determining the cell type identities and their distinctions (Fig. 3B; Supplemental Table 3). Consistent with its distinct cluster, 16 TF motifs were specifically accessible in BJ cells, such as FOS-JUN and NFE2, which were reported to display tissuespecific activities, especially in fibroblasts (Fig. 3B-D; Supplemental Fig. S6F; Wilkinson et al. 1989). On the other hand, BJ cells also extensively shared motifs with cells of myeloid lineage: K562 (79 motifs), including ETS1 and ZBTB33, and JK1 (28 motifs), including families of NFY, MEF2, and SP factors, compared with cells of lymphoid lineage-Jurkat (six motifs), including the TEAD family (Fig. 3B-D; Supplemental Fig. S6F). In addition, extensive overlap (46 motifs) was seen between cells of myeloid lineage (JK1 and K562), including TFs of GATA-TAL family (Fig. 3B-D). These enriched factors were reported to play important roles in the respective lineages. For instance, ZBTB33 is specifically important for K562 by binding to methylated DNA, whereas its depletion causes differentiation defects (Cofre et al. 2012; Lin et al. 2019). GATA1 is a master TF of erythropoiesis with specific expression in myeloid lineage but not in the lymphoblastic leukemia cells (Lee et al. 2017; Gutiérrez et al. 2020). On the contrary, inactivation of Hippo kinase modules, such as MST1/2, causes lymphopenia in human and results in translocation of YAP/TAZ to nucleus, which activates regulatory activity of TEAD TFs (Cheng et al. 2018; Yamauchi and Moroishi 2019). The gathered literature reports suggest inactive Hippo pathway in lymphoblast Jurkat cells and the resultant activation of TEAD activity.

Meanwhile, of 384 ASTAR RNA-seq libraries, 296 libraries (77.1\%) passed QC and showed full coverage for the expressed transcripts (Supplemental Fig. S6G-I; Supplemental Table 3). A total of 291 ASTAR-seq libraries (75.8\%) passed the QC filtration for both the scATAC-seq and scRNA-seq libraries (Supplemental Table 3). In addition, ASTAR-seq libraries showed high similarity $(R=$ 0.82 ) to the unimodal scRNA-seq libraries (Supplemental Fig. S6J; Pollen et al. 2014). Besides, transcriptomic profiles detected by ASTAR RNA-seq can distinguish among distinct cell lines with high accuracy (99.63\%) (Supplemental Fig. S6K). Furthermore, reference component analysis (RCA) analysis (Li et al. 2017) showed distinct correlation of $\mathrm{BJ}$ cells to foreskin fibroblasts and muscle lineage, JK1 cells to erythroblasts, K562 cells to leukemia K562, and Jurkat cells to leukemia lymphoblast (Fig. 3E). Consistent with their differential regulatory activities, GATA1 was uniquely accessible and expressed in myeloid JK1 and K562 cells, whereas SP1 was accessible and expressed in BJ, JK1, and K562 cells (Fig. 3F). Taken together, ASTAR-seq enables the identification of TFs responsible for the distinctions among distinct cell types.

\section{ASTAR-seq reveals dynamics in transcriptome and epigenome along the pseudotemporal axis of erythroblast differentiation}

To measure its capability in capturing the dynamic changes, we applied ASTAR-seq to the primary cells undergoing erythroblast differentiation. We harvested a total of 480 cells at day 6 (D6), day 8 (D8), day 10 (D10), and day12 (D12) of erythroblast differentiation, which was induced from mononuclear cells isolated from umbilical cord blood, for ASTAR-seq library preparation (Fig. 4A). Of the 480 ASTAR-seq libraries, 273 cells (56.9\%) presented good-quality libraries for both scATAC-seq and scRNA-seq (Supplemental Table 4). Comparatively, the QC rate is lower than that of the cell lines profiled earlier, which is likely associated with the inherent difficulty in culturing the primary cells. We next investigated the trajectories

\section{Genome Research}

www.genome.org 
A

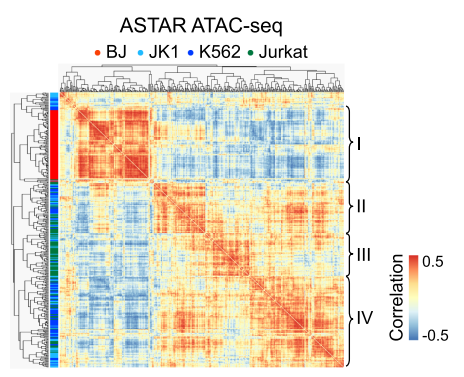

B

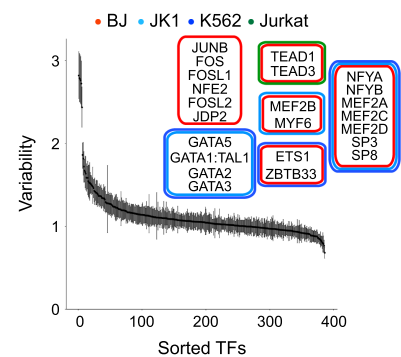

C

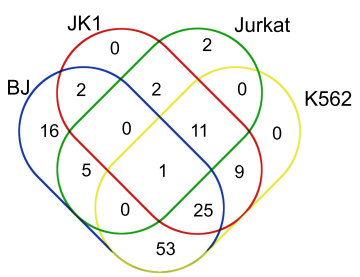

D ASTAR ATAC-seq
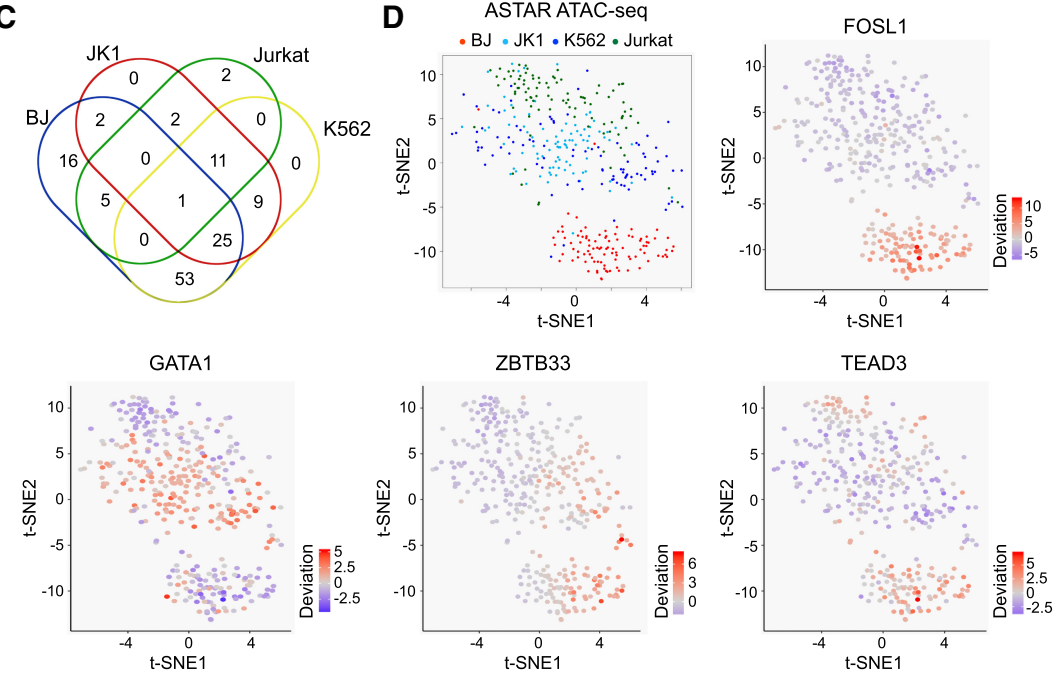

E
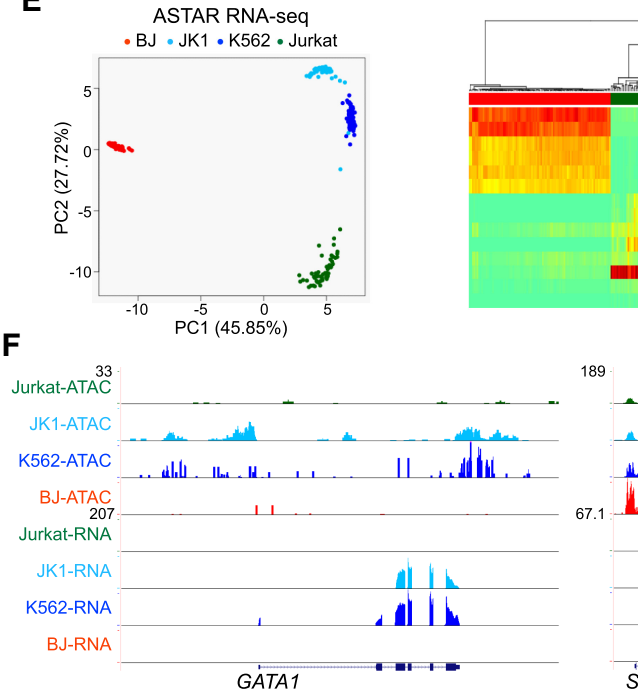

189

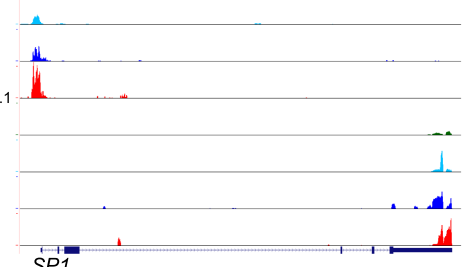

Figure 3. Application of ASTAR-seq on human cell lines. (A) Clustering of $B|| K 1,, K 562$, and Jurkat ASTAR ATAC-seq libraries based on the human JASPAR motif deviation scores calculated over the HARs. Color indicates the correlation level among the libraries, ranging from blue (no) to red (high). Side color bar ( $y$-axis) indicates the identity of each cell. $(B)$ Variability plot indicating the variable TF motifs across the ASTAR ATAC-seq libraries of four human cell lines. The $y$-axis represents the variability score assigned to each JASPAR motif, whereas the $x$-axis represents the motif rank. Top variable motifs are classified based on their enrichment scores across the cell lines and colored accordingly. (C) Multi-Venn diagram showing the shared and unique TFs across the cell lines. ( $D$, top left) t-SNE clustering of BJ, JK1, K562, and Jurkat ASTAR ATAC-seq libraries based on the deviation scores of human JASPAR motifs. Colors represent the cell lines. (Top right and bottom) Superimposition of motif enrichment scores for FOSL1, GATA1, ZBTB33, and TEAD3 on the t-SNE plot. Colors represent the motif enrichment levels, ranging from blue (no) to red (high). (E, left) PCA clustering of ASTAR RNA-seq libraries based on their correlation to the RCA panel. (Right) Heatmap showing the lineages that each cell correlates to. $(F)$ UCSC screenshots indicating the chromatin accessibility (top) and expression (bottom) levels of GATA1 (left) and SP1 (right) across the cell lines. of erythroblast differentiation using pseudotemporal analysis (Trapnell et al. 2014; Qiu et al. 2017). The resultant trajectories consisted of two branching events and five pseudotemporal states (Fig. 4B,C). Pseudotime highly correlated with the actual differentiation time points. For instance, cells of earlier time points, such as D6 and D8, were mostly at states $1-3$, whereas D10 cells were mostly at states 4 and 5 (Fig. 4B,C). On the other hand, the majority of D12 cells belonged to state 5 and located at the endpoint of pseudotime (Fig. 4B,C). Consistently, $H B A 2$, a hemoglobin gene, showed elevated expression in cells of state 5 compared with the others (Fig. 4B).

We then performed RCA analysis to examine the differentiation status of various pseudotemporal states. Generally, the majority of cells showed strong correlation to the myeloid or erythroid lineages (Fig. 4D). As the differentiation time point increased, transitions in the order of common myeloid progenitors (CMPs), myeloid erythroid progenitors (MEPs), erythroblast progenitors (Eryth pro), early erythroblasts, and late erythroblasts were observed (Fig. 4D). Specifically, the majority of states 1-3 cells showed a strong correlation to Eryth pro cells and certain degrees of correlation to MEPs and early erythroid (Fig. 4D). Cells of state 5 can be broadly classified into three groups, including groups with correlation to early erythroid but not to MEPs, with strong early erythroid identities, and with significant late erythroid fate (Fig. 4D). On the other hand, cells of state 4 bifurcated from the differentiation trajectory and acquired alternative fate resembling granulocyte monocyte progenitor (GMP) (Fig. 4D). In addition to this, GO analysis also showed that genes abundantly expressed in cells of state 5 were associated with oxygen transport, hydrogen peroxide catabolic process, and erythrocyte differentiation process, whereas state 4 cells highly expressed genes related to innate immune response and antigen processing and presentation (Supplemental Fig. S7A). On the contrary, compared with state 1 , genes that were repressed in state 5 were associated with stem cell population maintenance, positive regulation of $\mathrm{H3}$ K4 methylation, and chromatin organization, whereas genes related to erythrocyte maturation, oxygen transport, and NF-kB signaling were repressed in state 4 cells (Supplemental Fig. S7B). Altogether, 
A

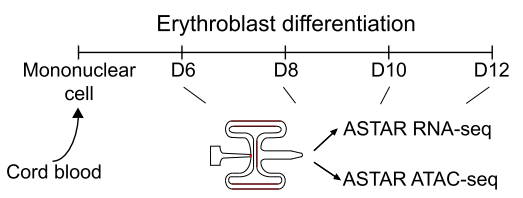

C
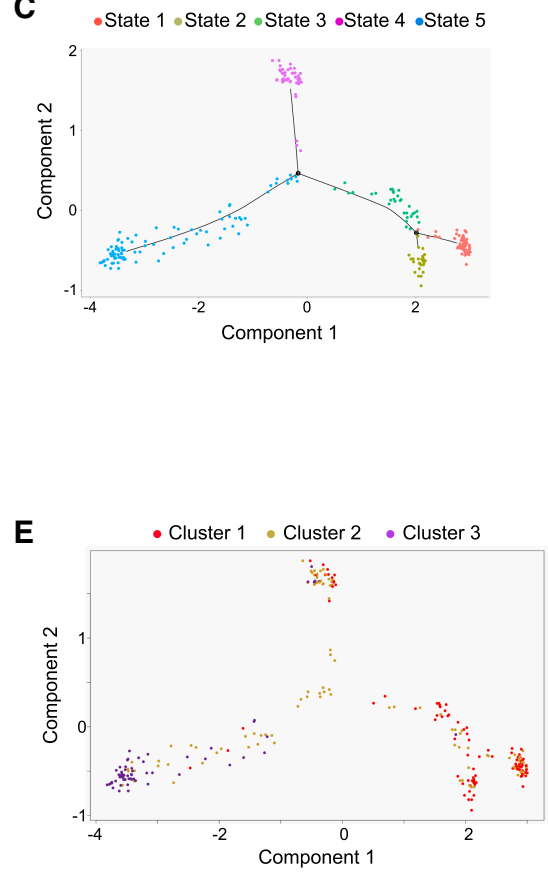

E

G

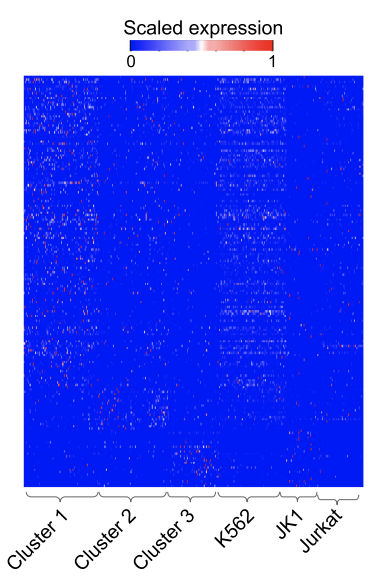

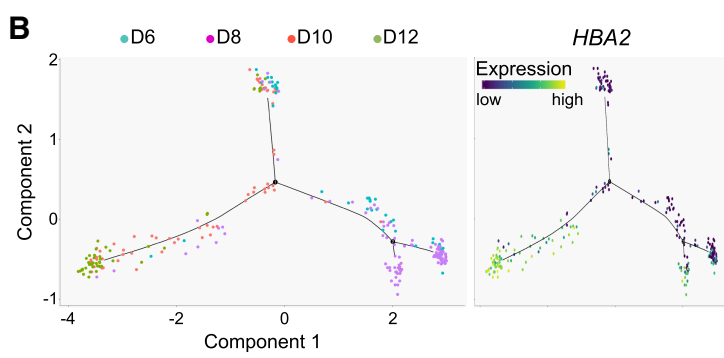

D

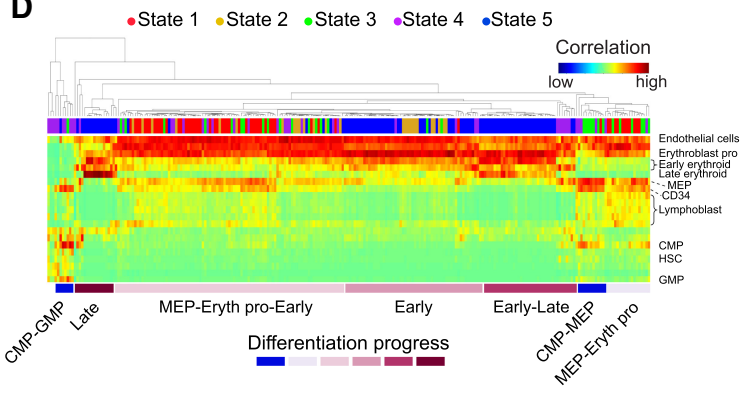

$\mathbf{F}$

H
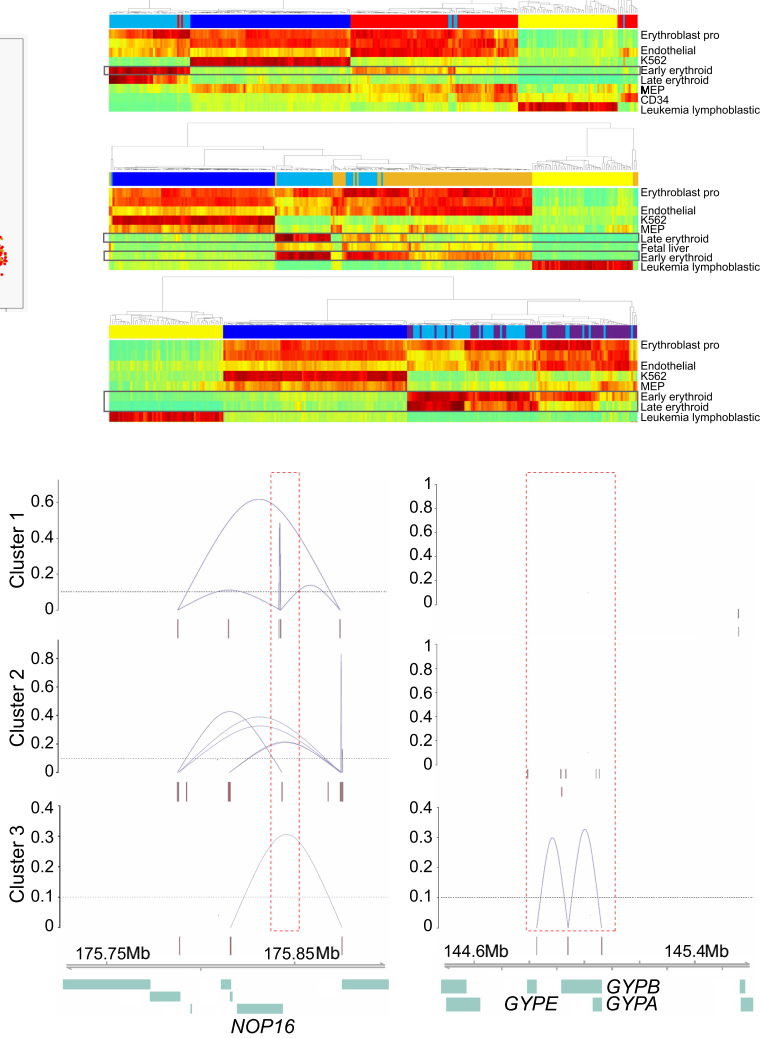

Figure 4. Interactive analysis of transcriptome and chromatin accessibility during erythroblast differentiation. (A) Schematic of erythroblast differentiation time points harvested for ASTAR-seq library preparation. ( $B$, left) Trajectory of erythroblast differentiation identified from ASTAR RNA-seq libraries using DDRTree dimension reduction. Colors represent time points. (Right) Superimposition of HBA2 expression on the trajectory. Colors represent expression levels. (C) Trajectory plot revealing the pseudotemporal states. Colors represent pseudotemporal states. (D) RCA heatmap showing the clustering of cells undergoing erythroblast differentiation, based on their correlation to the cells of different lineage origins in RCA panel. Color indicates correlation value, ranging from blue (low) to red (high). Each row indicates one lineage, whereas each column represents an ASTAR RNA-seq library. Pseudotemporal state of each cell is indicated on top. Cellular differentiation status is determined based on their correlation to the cells of RCA panel and indicated below. (E) Superimposition of NMF clusters on the trajectory plot. (F) RCA clustering of JK1, K562, and Jurkat cells with the cells of NMF cluster 1 (top), cluster 2 (middle), and cluster 3 (below), respectively. Color indicates correlation value, ranging from blue (low) to red (high). Each row indicates one lineage, whereas each column represents an ASTAR RNA-seq library. Cell identities are indicated on top. (G) Heatmap showing the expression of NMF cluster-specific genes across NMF clusters and the hematopoietic cell lines. Color indicates expression level, ranging from blue (no) to red (high). ( $H$ ) Line plots indicating the Cicero coaccessibility links between the regions highlighted in red and the distal sites in the surrounding regions. Height indicates the coaccessibility score of the connected peaks. The links are constructed from ASTAR ATAC-seq libraries of cluster 1 (top), cluster 2 (middle), and cluster 3 (bottom), respectively.

\section{Genome Research}

www.genome.org 
state 5 represents cells attaining erythroid fate, whereas state 4 cells deviate from erythroblast differentiation path, instead acquiring GMP identities.

To identify the genes and regulatory regions responsible for the progression of erythroblast differentiation, we used coupled NMF analysis, which uncovered three clusters (Fig. 4E). Superimposition of NMF clusters on the erythroblast differentiation trajectory showed that the majority of cluster 1 cells belonged to states 1-2, whereas cells of cluster 3 were the major constituent of state 5 (Fig. 4E). Cluster 2 cells scattered across the various states (Fig. 4E). Consistent with the cell fate of pseudotemporal states, cluster 1 cells showed characteristics of proerythroblasts and early erythroid cells and clustered closer to K562 cells, whereas cluster 2 cells showed stronger early erythroid identities and started to develop late erythroid characteristics (Fig. 4F). In addition, cluster 2 cells showed a higher similarity to JK1 than to K562 cells, reflecting their advanced differentiation compared with cluster 1 cells (Fig. 4F; Koeffler and Golde 1980), whereas cluster 3 cells displayed the strongest late erythroid identities and were clustered intimately with JK1 cells (Fig. 4F). Supporting this notion, differentially expressed genes of NMF cluster 1 were highly represented in K562 cells, whereas cluster 3 genes were specifically expressed in JK1 cells (Fig. 4G; Supplemental Table 4). This was further substantiated by CTen analysis (Supplemental Fig. S7C,D; Shoemaker et al. 2012). On the other hand, the accessibility of chromatins containing motif sequences of GATA1:TAL1 and MEF2D was highly variable across the NMF clusters (Supplemental Fig. S7E,F; Supplemental Table 4). In addition, cluster 1 accessible regions were highly enriched with HOXB4 and FOXA3 motifs, cluster 2 with ONECUT1 motif, and cluster 3 with POU2F2 and RUNX2 motifs, implicating the importance of these TFs in regulating the genes crucial for progression of erythroblast differentiation (Supplemental Fig. S7G; Supplemental Table 4). Moreover, the cluster-specific genes showed differential coaccessibility with its surrounding regulatory regions. For example, NOP16 was highly expressed and accessible in cluster 1 with multiple genomic interactions with the nearby genes, whereas the cluster 3-specific gene $G Y P B$ showed a high interaction frequency with GYPE only in cluster 3 (Fig. $4 \mathrm{H}$ ).

\section{Discussion}

In this study, we presented an automated bimodal single-cell technology, ASTAR-seq, which allows for parallel profiling of wholecell transcriptome and chromatin accessibility within the same single cell, with a greater sensitivity. Among the multimodal single-cell techniques for transcriptome and chromatin accessibility, sci-CAR, SNARE-seq, and Paired-seq are more suitable for organism-scale measurements, owing to their high-throughput profiling and low cost (Cao et al. 2018; Chen et al. 2019; Zhu et al. 2019). However, high-throughput profiling meanwhile results in a major drawback, massive loss of information, owing to the low sequencing depth. Apart from this, scRNA-seq libraries prepared by these high-throughput techniques specifically capture transcriptome within nucleus and are biased toward the $3^{\prime}$ end of mRNA. In sum, because of these drawbacks, high-throughput multimodal techniques are not suitable for studying lowly abundant transcripts, isoforms, and transcriptional maturation.

However, these could be achieved by using high-depth multimodal single-cell assays, such as scCAT-seq (Liu et al. 2019) and ASTAR-seq. scCAT-seq and ASTAR-seq display much greater detection sensitivity than the high-throughput multimodal techniques.
Comparatively, ASTAR-seq presents the following advantages compared with scCAT-seq. ASTAR-seq shows better performance in terms of genome alignment, gene detection sensitivity, and QC rate than scCAT-seq. In addition, ASTAR-seq measures transcriptomic profile within a whole cell, whereas scCAT-seq captures cytoplasmic transcriptome. Furthermore, owing to the integration with automated devices, ASTAR-seq displays higher consistency and requires less manual handling time and manpower. Therefore, the pros and cons of these multimodal assays should be carefully considered to better suit your application (Xing et al. 2020). Altogether, ASTAR-seq is a powerful integrated approach to understand the connectivity between transcription and epigenetic regulation.

\section{Methods}

\section{Cell culture}

mES-E14TG2a and 2i cells were cultured with the routinely used medium. DUX overexpressed mESCs was obtained following the previously described procedures (Hendrickson et al. 2017). mESCs were routinely propagated, passaged using trypsin, and replated onto $0.1 \%$ gelatin-coated plates every 3-4 d. BJ, K562, JK-1, and Jurkat were cultured with standard medium. Detailed medium recipes and induction methods can be found in the Supplemental Methods.

\section{ASTAR-seq}

Single-cell suspension was loaded onto Fluidigm C1 Open App microfluidic chips, and single-cell capture efficiency was assessed using a Nikon automated microscope. ASTAR-seq on-IFC steps were automatically performed on $\mathrm{C} 1$ machine using the custom built "ASTAR- ASTAR (1861x/1862x/1863x)" scripts. ASTAR-seq on-IFC reactions include lysis and transposition, EDTA (inactivate Tn5), $\mathrm{MgCl}_{2}$ (quench excess EDTA) and RT, and cDNA-PCR (biotinylation and amplification). Off-IFC steps are composed of streptavidin bead separation of cDNA and open chromatins (ATAC-DNA), PCR amplification of cDNA, and ATAC-seq and mRNA-seq library preparation. Detailed ASTAR-seq protocol can be found in the Supplemental Methods. ASTAR-seq scripts can be found in Supplemental Codes, ASTAR script.

\section{ASTAR-seq library sequencing}

One hundred ninety-two ASTAR RNA-seq libraries were sequenced in a lane of HiSeq 4000 sequencer using a 101-bp pair-end sequencing parameter. One hundred ninety-two ASTAR ATAC-seq libraries were sequenced in a lane of HiSeq 4000 sequencer using 50bp pair-end sequencing parameter.

\section{Mapping of ASTAR-seq libraries}

ASTAR-seq libraries were mapped to $\mathrm{mm} 9$ (for mouse libraries) and hg19 (for human libraries) using STAR aligner (Dobin et al. 2013; see Supplemental Methods). For scRNA-seq libraries, we allowed up to two mismatches and removed reads that map to more than one locus. The option "-outSAMstrandFiled intronMotif" was used to make BAM outputs of STAR compatible with subsequent analyses. For scATAC-seq libraries, options --alignIntronMax 1 and --alignEndsType EndToEnd were used. Detailed scripts can be found in Supplemental Code. ASTAR-seq library filtration criteria can be found in the Supplemental Methods. 


\section{Quantification and normalization of scRNA-seq libraries}

GTF files were generated using the "genePredToGtf" tool designed by UCSC. BAM files and generated GTF files were used as inputs for Cuffquant (Trapnell et al. 2010). Options - $u$ and $-m$ were included in the Cuffquant script. The abundances files were then used as inputs for Cuffnorm (Trapnell et al. 2010). The classic-fpkm normalization style was used. The detailed scripts can be found in Supplemental Code.

\section{Clustering of scRNA-seq libraries}

FPKM table was uploaded to Seurat (Butler et al. 2018) as input for t-SNE clustering. For UMAP generation, the raw counts generated by featureCounts were uploaded to Seurat v3. Data were then scaled, and variable genes were detected using default parameters. PCA was performed using the variable genes, and then UMAP or t-SNE were generated. The FeaturePlots function of Seurat was used to superimpose gene expression over UMAP plot. VlnPlot function was used to determine the number of genes detected per cell for all samples. The detailed script can be found in Supplemental Code.

\section{scRNA-seq analysis}

MCA (Han et al. 2018) and RCA (Li et al. 2017) analyses were performed as previously described. Details can be found in the Supplemental Methods, and the detailed script for RCA can be found in Supplemental Code.

\section{Pseudotime analysis}

FPKM table generated by Cuffnorm was used as an input for Monocle (Trapnell et al. 2014). FPKM values were converted to mRNA counts using the "relative2abs" function (Qiu et al. 2017). The expression family was set to negbinomial while creating the CellDataSet. Then, size factors and dispersions were estimated with default parameters. ReduceDimension function was used with method "DDRTree". This was followed by cell ordering (orderCells) and trajectory plotting. The detailed script can be found in Supplemental Code.

\section{Determination of HARs}

Human and mouse ASTAR ATAC-seq libraries were merged independently using SAMtools merge (Li et al. 2009). Duplicates were removed using the MarkDuplicates module of Picard (https ://broadinstitute.github.io/picard/). Peak calling was performed using MACS2 (Zhang et al. 2008), and the --nomodel --nolambda --keep-dup all --call-summits options were used. The narrowPeaks output of MACS2 was considered as the HARs. The detailed script can be found in Supplemental Code.

\section{chromVAR analysis}

scATAC-seq libraries were subjected to chromVAR analysis as previously described (Schep et al. 2017). Details can be found in the Supplemental Methods, and the detailed script can be found in Supplemental Code.

\section{Motif analysis}

The findMotifsGenome.pl script of HOMER (Heinz et al. 2010) was executed in order to identify the known motifs enriched in the differentially accessible regions.

\section{Integrative analysis}

Coupled NMF (Duren et al. 2018) was used to cluster the cells based on the integration of both scATAC-seq and scRNA-seq libraries, as previously described. Details and downstream analysis can be found in the Supplemental Methods. The detailed script can be found in Supplemental Code.

\section{Prediction of cis-regulatory interactions}

ASTAR ATAC-seq libraries belonging to each NMF cluster were merged using SAMtools merge to determine the HARs of each cluster as described above. Then coverage of each library belonging to a particular cluster over the HARs of its corresponding cluster was measured using DepthofCoverage as described above. The raw coverage table was used as an input for Cicero (Pliner et al. 2018). Cicero CDS were created using make_cicero_cds, and "run_ cicero" was then performed (with default settings). The plot_connections function was used to visualize the predicted regulatory elements of NMF-identified genes. Coordinates used were 10x zoomed-out from the precise coordinates of the genes of interest. The detailed script can be found in Supplemental Code.

\section{Interactome analysis}

Genes specific to each NMF cluster were uploaded to STRING (Szklarczyk et al. 2019). The text-mining option was disabled. The network formed was downloaded in tabular format, and then the tables were uploaded to Cytoscape (Shannon et al. 2003) for visual formatting.

\section{Confusion matrix generation}

The confusion matrixes were generated using MLSeq (Zararsiz et al. 2017). Details can be found in the Supplemental Methods, and the detailed script can be found in Supplemental Code.

\section{External data}

External data sets used in this study were downloaded from the following database under the specified accession numbers: BJ scRNA-seq (NCBI Sequence Read Archive [SRA]: SRP041736), K562 scRNA-seq (NCBI SRA: SRP041736), mESCs scRNA-seq (ArrayExpress database: E-MTAB-5484, E-MTAB-5485), BJ scAT AC-seq (NCBI Gene Expression Omnibus [GEO]: GSE65360), K562 scATAC-seq (GEO: GSE65360), mESCs scATAC-seq (GEO: GSE65360), scCAT-seq (CNGB Nucleotide Sequence Archive [CNSA]: CNP0000213).

\section{Data access}

All raw and processed sequencing data generated in this study have been submitted to the NCBI Gene Expression Omnibus (GEO; https://www.ncbi.nlm.nih.gov/geo/) under accession number GSE113418.

\section{Competing interest statement}

The authors declare no competing interests.

\section{Acknowledgments}

We thank Haitong Fang, Naresh Waran Gnanasegaran, and Sudhagar Samydurai for the technical assistance. H.L. is supported by National Instiutes of Health (AG056318, AG61796, and CA208517), the Glenn Foundation for Medical Research, Mayo

\section{Genome Research}

www.genome.org 
Clinic Center for Biomedical Discovery, Center for Individualized Medicine, Mayo Clinic Cancer Center, and the David F. and Margaret T. Grohne Cancer Immunology and Immunotherapy Program. Y.H.L. is supported by the National Research Foundation Investigatorship award (NRFI2018-02), Agency for Science, Technology and Research JCO Development Program Grant (1534n00153 and 1334k00083), and Singapore National Research Foundation under its Cooperative Basic Research grant administered by the Singapore Ministry of Health's National Medical Research Council (NMRC/CBRG/0092/2015). We thank the Biomedical Research Council, Agency for Science, Technology and Research, Singapore for research funding.

Author contributions: Q.R.X. developed the ASTAR-seq protocol, performed experiments, and wrote the paper. C.A.E.F. performed most of the bioinformatics analyses and wrote the paper; Y.Y.Z. performed part of the bioinformatics analyses; Y.Y., T.W., and P.G. assisted with the manuscript writing; J.J.C., J.X., P.D., C.G.K., H.L., and L.F.Z. analyzed data; and Y.H.L. designed the study, analyzed data, and wrote the paper.

\section{References}

Angermueller C, Clark SJ, Lee HJ, Macaulay IC, Teng MJ, Hu TX, Krueger F, Smallwood SA, Ponting CP, Voet T, et al. 2016. Parallel single-cell sequencing links transcriptional and epigenetic heterogeneity. Nat Methods 13: 229-232. doi:10.1038/nmeth.3728

Azzolin L, Panciera T, Soligo S, Enzo E, Bicciato S, Dupont S, Bresolin S, Frasson C, Basso G, Guzzardo V, et al. 2014. YAP/TAZ incorporation in the $\beta$-catenin destruction complex orchestrates the Wnt response. Cell 158: $157-170$. doi: $10.1016 / j$.cell.2014.06.013

Buecker C, Srinivasan R, Wu Z, Calo E, Acampora D, Faial T, Simeone A, Tan M, Swigut T, Wysocka J. 2014. Reorganization of enhancer patterns in transition from naive to primed pluripotency. Cell Stem Cell 14: 838853. doi:10.1016/j.stem.2014.04.003

Buenrostro JD, Wu B, Litzenburger UM, Ruff D, Gonzales ML, Snyder MP, Chang HY, Greenleaf WJ. 2015. Single-cell chromatin accessibility reveals principles of regulatory variation. Nature 523: 486-490. doi:10 $.1038 /$ nature 14590

Butler A, Hoffman P, Smibert P, Papalexi E, Satija R. 2018. Integrating singlecell transcriptomic data across different conditions, technologies, and species. Nat Biotechnol 36: 411-420. doi:10.1038/nbt.4096

Cao J, Cusanovich DA, Ramani V, Aghamirzaie D, Pliner HA, Hill AJ, Daza RM, McFaline-Figueroa JL, Packer JS, Christiansen L, et al. 2018. Joint profiling of chromatin accessibility and gene expression in thousands of single cells. Science 361: 1380-1385. doi:10.1126/science.aau0730

Chen Y, Lai D. 2015. Pluripotent states of human embryonic stem cells. Cell Reprogram 17: 1-6. doi:10.1089/cell.2014.0061

Chen H, Aksoy I, Gonnot F, Osteil P, Aubry M, Hamela C, Rognard C, Hochard A, Voisin S, Fontaine E, et al. 2015. Reinforcement of STAT3 activity reprogrammes human embryonic stem cells to naive-like pluripotency. Nat Commun 6: 7095. doi:10.1038/ncomms8095

Chen S, Lake BB, Zhang K. 2019. High-throughput sequencing of the transcriptome and chromatin accessibility in the same cell. Nat Biotechnol 37: 1452-1457. doi:10.1038/s41587-019-0290-0

Cheng J, Jing Y, Kang D, Yang L, Li J, Yu Z, Peng Z, Li X, Wei Y, Gong Q, et al. 2018. The role of Mst1 in lymphocyte homeostasis and function. Front Immunol 9: 149. doi:10.3389/fimmu.2018.00149

Cheow LF, Courtois ET, Tan Y, Viswanathan R, Xing Q, Tan RZ, Tan DSW, Robson P, Loh YH, Quake SR, et al. 2016. Single-cell multimodal profiling reveals cellular epigenetic heterogeneity. Nat Methods 13: 833-836. doi: $10.1038 /$ nmeth.3961

Choi J, Clement K, Huebner AJ, Webster J, Rose CM, Brumbaugh J, Walsh RM, Lee S, Savol A, Etchegaray JP, et al. 2017. DUSP9 modulates DNA hypomethylation in female mouse pluripotent stem cells. Cell Stem Cell 20: 706-719.e7. doi:10.1016/j.stem.2017.03.002

Clark SJ, Argelaguet R, Kapourani CA, Stubbs TM, Lee HJ, Alda-Catalinas C, Krueger F, Sanguinetti G, Kelsey G, Marioni JC, et al. 2018. scNMT-seq enables joint profiling of chromatin accessibility DNA methylation and transcription in single cells. Nat Commun 9: 781. doi:10.1038/ s41467-018-03149-4

Cofre J, Menezes JRL, Pizzatti L, Abdelhay E. 2012. Knock-down of Kaiso induces proliferation and blocks granulocytic differentiation in blast crisis of chronic myeloid leukemia. Cancer Cell Int 12: 28. doi:10.1186/14752867-12-28
Cusanovich DA, Daza R, Adey A, Pliner HA, Christiansen L, Gunderson KL, Steemers FJ, Trapnell C, Shendure J. 2015. Multiplex single-cell profiling of chromatin accessibility by combinatorial cellular indexing. Science 348: 910-914. doi:10.1126/science.aab1601

Dey SS, Kester L, Spanjaard B, Bienko M, Van Oudenaarden A. 2015. Integrated genome and transcriptome sequencing of the same cell. Nat Biotechnol 33: 285-289. doi:10.1038/nbt.3129

Dobin A, Davis CA, Schlesinger F, Drenkow J, Zaleski C, Jha S, Batut P, Chaisson M, Gingeras TR. 2013. STAR: ultrafast universal RNA-seq aligner. Bioinformatics 29: 15-21. doi:10.1093/bioinformatics/bts635

Duren Z, Chen X, Zamanighomi M, Zeng W, Satpathy AT, Chang HY, Wang Y, Wong WH. 2018. Integrative analysis of single-cell genomics data by coupled nonnegative matrix factorizations. Proc Natl Acad Sci 115: 7723-7728. doi:10.1073/pnas.1805681115

Flyamer IM, Gassler J, Imakaev M, Brandão HB, Ulianov SV, Abdennur N, Razin SV, Mirny LA, Tachibana-Konwalski K. 2017. Single-nucleus Hi$\mathrm{C}$ reveals unique chromatin reorganization at oocyte-to-zygote transition. Nature 544: 110-114. doi:10.1038/nature21711

Galan-Caridad JM, Harel S, Arenzana TL, Hou ZE, Doetsch FK, Mirny LA, Reizis B. 2007. Zfx controls the self-renewal of embryonic and hematopoietic stem cells. Cell 129: 345-357. doi:10.1016/j.cell.2007.03.014

Gangloff Y-G, Mueller M, Dann SG, Svoboda P, Sticker M, Spetz J-F, Um SH, Brown EJ, Cereghini S, Thomas G, et al. 2004. Disruption of the mouse mTOR gene leads to early postimplantation lethality and prohibits embryonic stem cell development. Mol Cell Biol 24: 9508-9516. doi:10 .1128/MCB.24.21.9508-9516.2004

Gawad C, Koh W, Quake SR. 2014. Dissecting the clonal origins of childhood acute lymphoblastic leukemia by single-cell genomics. Proc Natl Acad Sci 111: 17947-17952. doi:10.1073/pnas.1420822111

Ghimire S, Van Der Jeught M, Neupane J, Roost MS, Anckaert J, Popovic M, Van Nieuwerburgh F, Mestdagh P, Vandesompele J, Deforce D, et al. 2018. Comparative analysis of naive, primed and ground state pluripotency in mouse embryonic stem cells originating from the same genetic background. Sci Rep 8: 5884. doi:10.1038/s41598-018-24051-5

Grosselin K, Durand A, Marsolier J, Poitou A, Marangoni E, Nemati F, Dahmani A, Lameiras S, Reyal F, Frenoy O, et al. 2019. High-throughput single-cell ChIP-seq identifies heterogeneity of chromatin states in breast cancer. Nat Genet 51: 1060-1066. doi:10.1038/s41588-0190424-9

Guo G, Yang J, Nichols J, Hall JS, Eyres I, Mansfield W, Smith A. 2009. Klf4 reverts developmentally programmed restriction of ground state pluripotency. Development 136: 1063-1069. doi:10.1242/dev.030957

Guo H, Zhu P, Wu X, Li X, Wen L, Tang F. 2013. Single-cell methylome landscapes of mouse embryonic stem cells and early embryos analyzed using reduced representation bisulfite sequencing. Genome Res 23: 21262135. doi:10.1101/gr.161679.113

Gutiérrez L, Caballero N, Fernández-Calleja L, Karkoulia E, Strouboulis J. 2020. Regulation of GATA1 levels in erythropoiesis. IUBMB Life 72: 89-105. doi:10.1002/iub.2192

Han DW, Greber B, Wu G, Tapia N, Araúzo-Bravo MJ, Ko K, Bernemann C, Stehling M, Schöler HR. 2011. Direct reprogramming of fibroblasts into epiblast stem cells. Nat Cell Biol 13: 66-71. doi:10.1038/ncb2136

Han X, Wang R, Zhou Y, Fei L, Sun H, Lai S, Saadatpour A, Zhou Z, Chen H, Ye F, et al. 2018. Mapping the mouse cell atlas by microwell-seq. Cell 172: 1091-1107.e17. doi:10.1016/j.cell.2018.02.001

Hashimoto M, Sasaki H. 2019. Epiblast formation by TEAD-YAP-dependent expression of pluripotency factors and competitive elimination of un specified cells. Dev Cell 50: 139-154.e5. doi:10.1016/j.devcel.2019.05 .024

Heinz S, Benner C, Spann N, Bertolino E, Lin YC, Laslo P, Cheng JX, Murre C, Singh H, Glass CK. 2010. Simple combinations of lineage-determining transcription factors prime cis-regulatory elements required for macrophage and B cell identities. Mol Cell 38: 576-589. doi:10.1016/j molcel.2010.05.004

Hendrickson PG, Doráis JA, Grow EJ, Whiddon JL, Lim JW, Wike CL, Weaver BD, Pflueger C, Emery BR, Wilcox AL, et al. 2017. Conserved roles of mouse DUX and human DUX4 in activating cleavage-stage genes and MERVL/HERVL retrotransposons. Nat Genet 49: 925-934. doi:10.1038/ng.3844

Hu Y, Huang K, An O, Du G, Hu G, Xue J, Zhu X, Wang CY, Xue Z, Fan G. 2016. Simultaneous profiling of transcriptome and DNA methylome from a single cell. Genome Biol 17: 88. doi:10.1186/s13059-016-0950-z

Huang B, Wu H, Bhaya D, Grossman A, Granier S, Kobilka BK, Zare RN. 2007. Counting low-copy number proteins in a single cell. Science 315: 81-84. doi:10.1126/science. 1133992

Hughes AJ, Spelke DP, Xu Z, Kang C-C, Schaffer DV, Herr AE. 2014. Singlecell western blotting. Nat Methods 11: 749-755. doi:10.1038/nmeth .2992

Jeon H, Waku T, Azami T, Khoa LTP, Yanagisawa J, Takahashi S, Ema M. 2016. Comprehensive identification of Krüppel-like factor family members contributing to the self-renewal of mouse embryonic stem cells and 
cellular reprogramming. PLoS One 11: e0150715. doi:10.1371/journal .pone.0150715

Jin W, Tang Q, Wan M, Cui K, Zhang Y, Ren G, Ni B, Sklar J, Przytycka TM, Childs R, et al. 2015. Genome-wide detection of DNase I hypersensitive sites in single cells and FFPE tissue samples. Nature 528: 142-146. doi:10 $.1038 /$ nature 15740

Klein AM, Mazutis L, Akartuna I, Tallapragada N, Veres A, Li V, Peshkin L, Weitz DA, Kirschner MW. 2015. Droplet barcoding for single-cell transcriptomics applied to embryonic stem cells. Cell 161: 1187-1201. doi:10.1016/i.cell.2015.04.044

Koeffler H, Golde D. 1980. Human myeloid leukemia cell lines: a review. Blood 56: 344-350. doi:10.1182/blood.V56.3.344.344

Krijger PHL, Di Stefano B, De Wit E, Limone F, Van Oevelen C, De Laat W, Graf T. 2016. Cell-of-origin-specific 3D genome structure acquired during somatic cell reprogramming. Cell Stem Cell 18: 597-610. doi:10 $.1016 /$ j.stem.2016.01.007

Lee WY, Weinberg OK, Pinkus GS. 2017. GATA1 is a sensitive and specific nuclear marker for erythroid and megakaryocytic lineages. Am J Clin Pathol 147: 420-426. doi:10.1093/ajcp/aqx018

Leitch HG, Mcewen KR, Turp A, Encheva V, Carroll T, Grabole N, Mansfield W, Nashun B, Knezovich JG, Smith A, et al. 2013. Naive pluripotency is associated with global DNA hypomethylation. Nat Struct Mol Biol 20: 311-316. doi:10.1038/nsmb. 2510

Li H, Handsaker B, Wysoker A, Fennell T, Ruan J, Homer N, Marth G, Abecasis G, Durbin R, 1000 Genome Project Data Processing Subgroup. 2009. The Sequence Alignment/Map format and SAMtools. Bioinformatics 25: 2078-2079. doi:10.1093/bioinformatics/btp352

Li H, Courtois ET, Sengupta D, Tan Y, Chen KH, Goh JJL, Kong SL, Chua C, Hon LK, Tan WS, et al. 2017. Reference component analysis of singlecell transcriptomes elucidates cellular heterogeneity in human colorectal tumors. Nat Genet 49: 708-718. doi:10.1038/ng.3818

Lian I, Kim J, Okazawa H, Zhao J, Zhao B, Yu J, Chinnaiyan A, Israel MA Goldstein LSB, Abujarour R, et al. 2010. The role of YAP transcription coactivator in regulating stem cell self-renewal and differentiation. Genes Dev 24: 1106-1118. doi:10.1101/gad.1903310

Lin QXX, Rebbani K, Jha S, Benoukraf T. 2019. ZBTB33 (Kaiso) methylated binding sites are associated with primed heterochromatin. bioRxiv doi:10.1101/585653

Liu L, Liu C, Quintero A, Wu L, Yuan Y, Wang M, Cheng M, Leng L, Xu L, Dong G, et al. 2019. Deconvolution of single-cell multi-omics layers reveals regulatory heterogeneity. Nat Commun 10: 470. doi:10.1038/ s41467-018-08205-7

Lorthongpanich C, Cheow LF, Balu S, Quake SR, Knowles BB, Burkholder WF, Solter D, Messerschmidt DM. 2013. Single-cell DNA-methylation analysis reveals epigenetic chimerism in preimplantation embryos. Science 341: 1110-1112. doi:10.1126/science.1240617

Macaulay IC, Haerty W, Kumar P, Li YI, Hu TX, Teng MJ, Goolam M, Saurat N, Coupland P, Shirley LM, et al. 2015. G\&T-seq: parallel sequencing of single-cell genomes and transcriptomes. Nat Methods 12: 519-522. doi:10.1038/nmeth.3370

Macfarlan TS, Gifford WD, Driscoll S, Lettieri K, Rowe HM, Bonanomi D Firth A, Singer O, Trono D, Pfaff SL. 2012. Embryonic stem cell potency fluctuates with endogenous retrovirus activity. Nature 487: 57-63. doi:10.1038/nature 11244

Macosko EZ, Basu A, Satija R, Nemesh J, Shekhar K, Goldman M, Tirosh I, Bialas AR, Kamitaki N, Martersteck EM, et al. 2015. Highly parallel genome-wide expression profiling of individual cells using nanoliter droplets. Cell 161: 1202-1214. doi:10.1016/j.cell.2015.05.002

Mathieu J, Ruohola-Baker H. 2017. Metabolic remodeling during the loss and acquisition of pluripotency. Development 144: 541-551. doi:10 $.1242 /$ dev.128389

Nagano T, Lubling Y, Stevens TJ, Schoenfelder S, Yaffe E, Dean W, Laue ED, Tanay A, Fraser P. 2013. Single-cell Hi-C reveals cell-to-cell variability in chromosome structure. Nature 502: 59-64. doi:10.1038/nature12593

Navin N, Kendall J, Troge J, Andrews P, Rodgers L, McIndoo J, Cook K, Stepansky A, Levy D, Esposito D, et al. 2011. Tumour evolution inferred by single-cell sequencing. Nature 472: $90-94$. doi:10.1038/nature09807

Pliner HA, Packer JS, McFaline-Figueroa JL, Cusanovich DA, Daza RM, Aghamirzaie D, Srivatsan S, Qiu X, Jackson D, Minkina A, et al. 2018. Cicero predicts cis-regulatory DNA interactions from single-cell chromatin accessibility data. Mol Cell 71: 858-871.e8. doi:10.1016/j.molcel .2018 .06 .044

Pollen AA, Nowakowski TJ, Shuga J, Wang X, Leyrat AA, Lui JH, Li N, Szpankowski L, Fowler B, Chen P, et al. 2014. Low-coverage single-cell mRNA sequencing reveals cellular heterogeneity and activated signaling pathways in developing cerebral cortex. Nat Biotechnol 32: 1053-1058. doi:10.1038/nbt.2967

Pott S. 2017. Simultaneous measurement of chromatin accessibility, DNA methylation, and nucleosome phasing in single cells. eLife 6: e23203. doi:10.7554/eLife.23203
Qiu X, Mao Q, Tang Y, Wang L, Chawla R, Pliner HA, Trapnell C. 2017. Reversed graph embedding resolves complex single-cell trajectories. Nat Methods 14: 979-982. doi:10.1038/nmeth.4402

Raj B, Wagner DE, McKenna A, Pandey S, Klein AM, Shendure J, Gagnon JA, Schier AF. 2018. Simultaneous single-cell profiling of lineages and cell types in the vertebrate brain. Nat Biotechno 36: 442-450. doi:10.1038/ nbt. 4103

Ramani V, Deng X, Qiu R, Gunderson KL, Steemers FJ, Disteche CM, Noble WS, Duan Z, Shendure J. 2017. Massively multiplex single-cell Hi-C. Nat Methods 14: 263-266. doi:10.1038/nmeth.4155

Rotem A, Ram O, Shoresh N, Sperling RA, Goren A, Weitz DA, Bernstein BE. 2015. Single-cell ChIP-seq reveals cell subpopulations defined by chromatin state. Nat Biotechnol 33: 1165-1172. doi:10.1038/nbt.3383

Sang H, Wang D, Zhao S, Zhang J, Zhang Y, Xu J, Chen X, Nie Y, Zhang K, Zhang S, et al. 2019. Dppa3 is critical for Lin28a-regulated ES cells naïveprimed state conversion. J Mol Cell Biol 11: 474-488. doi:10.1093/jmcb/ mjy069

Schep AN, Wu B, Buenrostro JD, Greenleaf WJ. 2017. chromVAR: inferring transcription-factor-associated accessibility from single-cell epigenomic data. Nat Methods 14: 975-978. doi:10.1038/nmeth.4401

Shannon P, Markiel A, Ozier O, Baliga NS, Wang JT, Ramage D, Amin N, Schwikowski B, Ideker T. 2003. Cytoscape: a software environment for integrated models of biomolecular interaction networks. Genome Res 13: 2498-2504. doi:10.1101/gr.1239303

Shoemaker JE, Lopes TJS, Ghosh S, Matsuoka Y, Kawaoka Y, Kitano H. 2012. CTen: a web-based platform for identifying enriched cell types from heterogeneous microarray data. BMC Genomics 13: 460. doi:10.1186/14712164-13-460

Smallwood SA, Lee HJ, Angermueller C, Krueger F, Saadeh H, Peat J, Andrews SR, Stegle O, Reik W, Kelsey G. 2014. Single-cell genome-wide bisulfite sequencing for assessing epigenetic heterogeneity. Nat Methods 11: 817-820. doi:10.1038/nmeth.3035

Stoeckius M, Hafemeister C, Stephenson W, Houck-Loomis B, Chattopadhyay PK, Swerdlow H, Satija R, Smibert P. 2017. Simultaneous epitope and transcriptome measurement in single cells. Nat Methods 14: 865-868. doi:10.1038/nmeth.4380

Svensson V, Natarajan KN, Ly LH, Miragaia RJ, Labalette C, Macaulay IC, Cvejic A, Teichmann SA. 2017. Power analysis of single-cell RNA-sequencing experiments. Nat Methods 14: 381-387. doi:10.1038/nmeth 4220

Szklarczyk D, Gable AL, Lyon D, Junge A, Wyder S, Huerta-Cepas J, Simonovic M, Doncheva NT, Morris JH, Bork P, et al. 2019. STRING v11: protein-protein association networks with increased coverage, supporting functional discovery in genome-wide experimental datasets. Nucleic Acids Res 47: D607-D613. doi:10.1093/nar/gky1131

Taleahmad S, Mirzaei M, Parker LM, Hassani SN, Mollamohammadi S, Sharifi-Zarchi A, Haynes PA, Baharvand H, Salekdeh GH. 2015. Proteome analysis of ground state pluripotency. Sci Rep 5: 17985. doi:10.1038/srep17985

Taleahmad S, Hassani SN, Salekdeh GH, Baharvand H. 2018. Metabolic signature of pluripotent stem cells. Cell J 20: 388-395. doi:10.22074/cellj .2018 .5514

Tang F, Barbacioru C, Wang Y, Nordman E, Lee C, Xu N, Wang X, Bodeau J, Tuch BB, Siddiqui A, et al. 2009. mRNA-Seq whole-transcriptome analysis of a single cell. Nat Methods 6: 377-382. doi:10.1038/nmeth.1315

Tang F, Barbacioru C, Nordman E, Li B, Xu N, Bashkirov VI, Lao K, Surani MA. 2010. RNA-Seq analysis to capture the transcriptome landscape of a single cell. Nat Protoc 5: 516-535. doi:10.1038/nprot.2009.236

Ter Huurne M, Chappell J, Dalton S, Stunnenberg HG. 2017. Distinct cellcycle control in two different states of mouse pluripotency. Cell Stem Cell 21: 449-455.e4. doi:10.1016/j.stem.2017.09.004

Trapnell C, Williams BA, Pertea G, Mortazavi A, Kwan G, Van Baren MJ, Salzberg SL, Wold BJ, Pachter L. 2010. Transcript assembly and quantification by RNA-Seq reveals unannotated transcripts and isoform switching during cell differentiation. Nat Biotechnol 28: 511-515. doi:10.1038/nbt.1621

Trapnell C, Cacchiarelli D, Grimsby J, Pokharel P, Li S, Morse M, Lennon NJ, Livak KJ, Mikkelsen TS, Rinn JL. 2014. The dynamics and regulators of cell fate decisions are revealed by pseudotemporal ordering of single cells. Nat Biotechnol 32: 381-386. doi:10.1038/nbt.2859

Wang J, Fan HC, Behr B, Quake SR. 2012. Genome-wide single-cell analysis of recombination activity and de novo mutation rates in human sperm. Cell 150: 402-412. doi:10.1016/j.cell.2012.06.030

Wang Q, Xiong H, Ai S, Yu X, Liu Y, Zhang J, He A. 2019. CoBATCH for highthroughput single-cell epigenomic profiling. Mol Cell 76: 206-216.e7. doi:10.1016/j.molcel.2019.07.015

Weinberger L, Ayyash M, Novershtern N, Hanna JH. 2016. Dynamic stem cell states: naive to primed pluripotency in rodents and humans. Nat Rev Mol Cell Biol 17: 155-169. doi:10.1038/nrm.2015.28 
Wilkinson DG, Bhatt S, Ryseck RP, Bravo R. 1989. Tissue-specific expression of c-jun and junB during organogenesis in the mouse. Development 106: $465-471$.

Xing QR, Cipta NO, Hamashima K, Liou YC, Koh CG, Loh YH. 2020. Unraveling heterogeneity in transcriptome and its regulation through single-cell multi-omics technologies. Front Genet 11: 662. doi:10.3389/ fgene.2020.00662

Yamauchi T, Moroishi T. 2019. Hippo pathway in mammalian adaptive immune system. Cells 8: 398. doi:10.3390/cells8050398

Ye S, Zhang D, Cheng F, Wilson D, Mackay J, He K, Ban Q, Lv F, Huang S, Liu $D$, et al. 2016. Wnt/ $\beta$-catenin and LIF-Stat3 signaling pathways converge on Sp5 to promote mouse embryonic stem cell self-renewal. J Cell Sci 129: 269-276. doi:10.1242/jcs.177675

Yu JSL, Cui W. 2016. Proliferation, survival and metabolism: the role of $\mathrm{PI} 3 \mathrm{~K} / \mathrm{AKT} / \mathrm{mTOR}$ signalling in pluripotency and cell fate determination. Development 143: 3050-3060. doi:10.1242/dev.137075

Zararsiz G, Goksuluk D, Korkmaz S, Eldem V, Zararsiz GE, Duru IP, Ozturk A. 2017. A comprehensive simulation study on classification of
RNA-Seq data. PLoS One 12: e0182507. doi:10.1371/journal.pone .0182507

Zhang Y, Liu T, Meyer CA, Eeckhoute J, Johnson DS, Bernstein BE, Nusbaum C, Myers RM, Brown M, Li W, et al. 2008. Model-based Analysis of ChIP-Seq (MACS). Genome Biol 9: R137. doi:10.1186/gb2008-9-9-r137

Zhu C, Yu M, Huang H, Juric I, Abnousi A, Hu R, Lucero J, Behrens MM, Hu M, Ren B. 2019. An ultra high-throughput method for single-cell joint analysis of open chromatin and transcriptome. Nat Struct Mol Biol 26: 1063-1070. doi:10.1038/s41594-019-0323-x

Zong C, Lu S, Chapman AR, Xie XS. 2012. Genome-wide detection of singlenucleotide and copy-number variations of a single human cell. Science 338: 1622-1626. doi:10.1126/science.1229164

Received October 2, 2019; accepted in revised form June 25, 2020. 


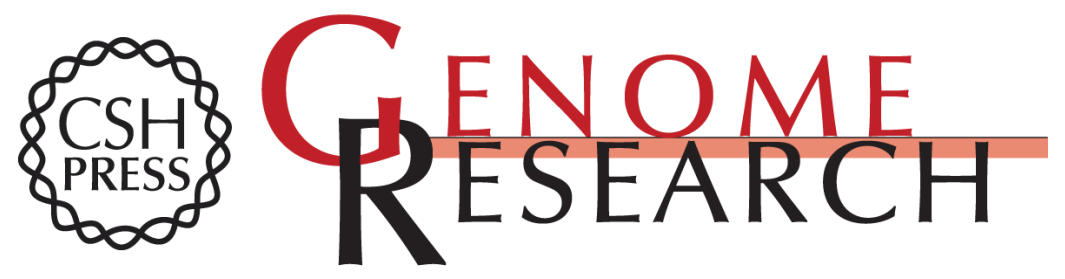

\section{Parallel bimodal single-cell sequencing of transcriptome and chromatin accessibility}

Qiao Rui Xing, Chadi A El Farran, Ying Ying Zeng, et al.

Genome Res. 2020 30: 1027-1039 originally published online July 22, 2020

Access the most recent version at doi:10.1101/gr.257840.119

Supplemental Material

References

Open Access

Creative Commons

License

Email Alerting Service
http://genome.cshlp.org/content/suppl/2020/07/22/gr.257840.119.DC1

This article cites 88 articles, 18 of which can be accessed free at: http://genome.cshlp.org/content/30/7/1027.full.html\#ref-list-1

Freely available online through the Genome Research Open Access option.

This article, published in Genome Research, is available under a Creative Commons License (Attribution-NonCommercial 4.0 International), as described at http://creativecommons.org/licenses/by-nc/4.0/.

Receive free email alerts when new articles cite this article - sign up in the box at the top right corner of the article or click here.

\section{Affordable, Accurate Sequencing.}

